02

\title{
Электронное строение и спектрально-флуоресцентные свойства лазерных красителей тиопирило-4-трикарбоцианинов
}

\author{
(C) А.А. Ищенко ${ }^{1}$, И.В. Курдюкова ${ }^{1}$, М.В. Богданович ${ }^{2}$, С.Л. Бондарев ${ }^{2}$, А.А. Романенко ${ }^{2}$, А.Г. Рябцев ${ }^{2, \text {, }}$ \\ Г.И. Рябцев ${ }^{2}$ \\ ${ }^{1}$ Институт органической химии НАН Украины, \\ 02660 Киев, Украина \\ e-mail: al.al.ishchenko@gmail.com \\ ${ }^{2}$ Институт фризики им. Б.И. Степанова НАН Беларуси, \\ 220070 Минск, Беларусь \\ e-mail: al.al.ishchenko@gmail.com, `ryabtsev@ifanbel.bas-net.by
}

Поступила в редакцию 11.02.2021 г.

В окончательной редакции 11.02.2021 г.

Принята к публикации 02.04.2021 г.

\begin{abstract}
Обнаружено, что длинноволновая полоса поглощения лазерного красителя IR 1061 и его аналога с незамещенной полиметиновой цепью сильно уширяется и уменьшается по интенсивности в полярных растворителях, тогда как полоса флуоресценции остается узкой и практически не изменяется в широком диапазоне полярности растворителей. На основании квантово-химических расчетов этих красителей неэмпирическим методом DFT/B3LYP/6-31G(d,p) и TDDFT с учетом полярности среды методом РCM показано, что причиной такого различия является ослабление сольватации в флуоресцентном состоянии по сравнению с основным вследствие большей выравненности заряда в первом, чем в последнем. Обнаружено увеличение альтернации порядков связей в полиметиновой цепи в флуоресцентном состоянии, что обусловливает усиление вибронных взаимодействий в излучательном переходе по сравнению с поглощательным. Проанализированы спектральные эффекты, вызванные изменением угла поворота фенильных групп в тиопирилиевом цикле при возбуждении.
\end{abstract}

Ключевые слова: тиопирило-4-трикарбоцианины, спектрально-люминесцентные свойства, сольватация, DFT/B3LYP/6-31G(d,p)- и TDDFT-расчеты, метод PCM.

DOI: $10.21883 /$ OS.2021.07.51077.1902-21

\section{Введение}

Полиметиновые красители вследствие наиболее широкого диапазона изменения спектрально-люминесцентных и нелинейно-оптических свойств среди органических красителей используются для решения разнообразных задач науки и техники [1-5]. Одной из важнейших таких задач является разработка лазерных сред на их основе, особенно в ближнем ИК диапазоне спектра [3]. В этом диапазоне спектра весьма популярными являются полиметины - производные тиопирилия (тиопирилоцианины) [6,7]. Один из них - мезо-хлорзамещенный тиопирило-4-трикарбоцианин с триметиленовой мостиковой группировкой в полиметиновой цепи, краситель 1 широко известен как IR 1061 и обладает уникальным сочетанием модуляционных и генерационных свойств. Как пассивный затвор твердотельных неодимовых лазеров он обеспечивает высокий уровень модуляции добротности и степени просветления [7]. Впервые на нем была получена генерация в ближнем ИК диапазоне при накачке длиной волны $1060 \mathrm{~nm}$ полимерной активной лазерной среды [8]. Причем эффективность генерации сравнима с органическими активными средами видимого спектрального диапазона $[9,10]$. Однако особенности спектральнолюминесцентных свойств красителя 1 (схема) и их связь с его электронным строением практически не исследованы. Основное внимание уделялось изучению влияния природы растворителя на квантовый выход флуоресценции и проблемам корректного его определения в ближнем ИК диапазоне $[11,12]$.

Целью настоящей работы явилось исследование электронного строения и спектрально-люминесцентных свойств красителя $\mathbf{1}$ в сравнении с его аналогом с незамещенной полиметиновой цепью - тиопирило-4-трикарбоцианином 2 (схема).<smiles>ClC1=C(/C=C/c2cc(-c3ccccc3)[s+]c(-c3ccccc3)c2)CCC/C1=C\C=C1C=C(c2ccccc2)SC(c2ccccc2)=C1</smiles>

1<smiles></smiles> 


\section{Методика эксперимента}

Синтез красителей 1 и 2 описан в [2] и [13] соответственно. В работе использовались растворители Sigma Aldrich (США). Спектры поглощения красителей регистрировались на спектрофотометре Cary-500 (США), а спектры фотолюминесценции растворов - на спектрофлуориметре Fluorolog-3 (HORIBA Scientific, США). В качестве детектора в люминесцентных измерениях использовалась охлаждаемая жидким азотом InGaAs ПЗС-линейка Symphony II (HORIBA Scientific). Спектры фотолюминесценции скорректированы на спектральную чувствительность прибора.

Квантово-химические расчеты электронного строения и энергий переходов красителей $\mathbf{1}$ и $\mathbf{2}$ выполнены неэмпирическими методами DFT и TD-DFT соответственно в программном пакете Gaussian-09 с использованием гибридного В3LYР-функционала [14]. Базис 6-31G(d,p) выбран как разумный компромисс скорости и точности [15]. Геометрия основного состояния в полностью транс-конформации красителей оптимизирована в дихлорометане (ДХМ), так как основные экспериментальные данные получены в этом растворителе. Производился расчет колебательной задачи, чтобы убедиться, что найденные стационарные точки являются истинными минимумами. Об этом свидетельствует отсутствие отрицательных (виртуальных) частот в колебательной задаче. Влияние растворителя рассчитывалось наиболее распространенным на сегодня методом моделирования действия растворителя - методом самосогласованного реакционного поля (SCRF), который в популярных квантово-химических пакетах реализован с помощью integral equation formalism variant of Polarizable Continuum Model (IEFPCM, далее PCM) [16]. Растворитель в рамках этого подхода рассматривается как непрерывная поляризованная среда (polarizable continuum), а полость модельной молекулы формируется набором сфер, которые перекрываются. Полярность растворителя задается ограниченным набором параметров: статической диэлектрической проницаемостью $\varepsilon_{D}$, динамической диэлектрической проницаемостью (пропорциональной коэффициенту преломления $n_{D}$ ) и средним радиусом молекулы растворителя [16].

Известно, что энергия длинноволнового электронного перехода, рассчитанная методом TD-DFT, в полиметиновых красителях различной ионности обычно превышает экспериментально определенную [17]. Причем учет влияния сольватации несущественно улучшает согласование расчета и эксперимента [17]. В качестве частичного решения этой проблемы в работах [18] предложено использование линейных корреляционных уравнений, полученных путем регрессионного анализа расчетных и экспериментальных максимумов поглощения для большого набора катионных полиметинов. Поэтому в значения рассчитанных максимумов красителей $\mathbf{1}$ и $\mathbf{2}$ внесены поправки в соответствии с данными работы [18].
Расчет порядков связей и зарядов на атомах в молекулах выполнен в рамках модели натуральных связывающих орбиталей (NBO, natural bond orbitals) с помощью встроенного в Gaussian-09 алгоритма [19].

Анализ электронной плотности проведен с помощью Multiwfn 3.7 [18]. Визуализация - программой Chemcraft 1.8.

Расчеты проведены с использованием вычислительных ресурсов объединенного вычислительного кластера НТК „Институт монокристаллов“ и Института сцинтилляционных материалов НАН Украины в составе Украинского национального грида.

\section{Результаты и их обсуждение}

Экспериментальные и рассчитанные спектральнолюминесцентные характеристики красителей 1 и 2 приведены в табл. 1.

Краситель 1 и 2 в ДХМ имеет характерную для типичных органических красителей универсальную форму длинноволновой полосы поглощения [1]. Она асимметрична за счет пологого коротковолнового подъема и резкого спада на длинноволновом краю (рис. 1) и в ее коротковолновой области отчетливо проявляется перегиб с интенсивностью, значительно меньшей, чем основной максимум. Первый отстоит на расстоянии $1200-1300 \mathrm{~cm}^{-1}$ от последнего. Эта величина соответствует частоте валентного полносимметричного колебания углеродных атомов хромофора в возбужденном состоянии красителя, которое отвечает за вибронную структуру длинноволнового электронного перехода [1]. Еще более строгим доказательством того, что коротковолновый максимум имеет колебательную природу, является зеркальное подобие спектров поглощения и флуоресценции, как видно на рис. 2 на примере красителя 1 в ДХМ.

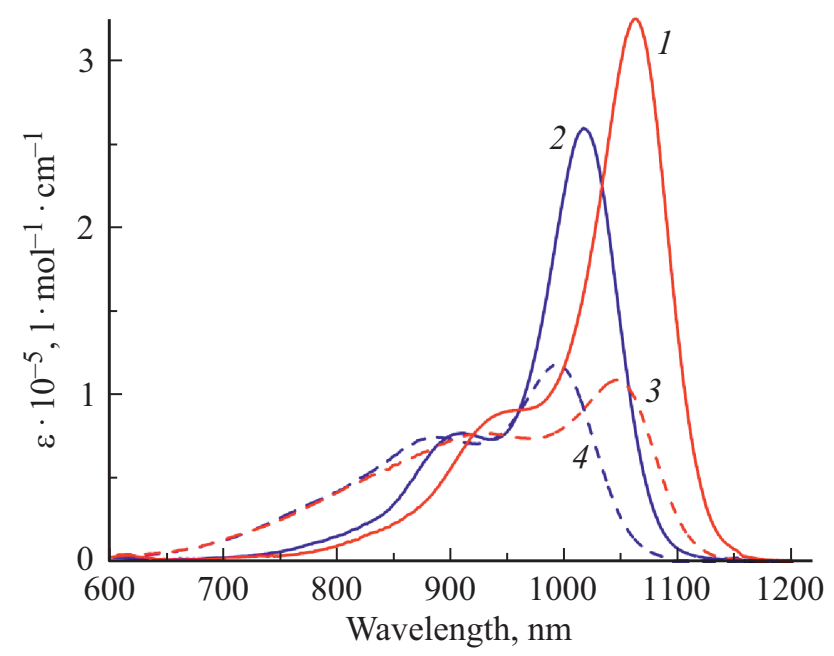

Рис. 1. Электронные спектры поглощения красителей $1(1,3)$ и $2(2,4)$ в дихлорометане $(1,2)$ и ацетонитриле $(3,4)$. 
Таблица 1. Значения экспериментальных максимумов поглощения $\lambda_{\mathrm{abs}}^{\exp }$ и флуоресценции $\lambda_{\mathrm{fl}}^{\text {exp }}$ молярных экстинкций $\varepsilon$ в дихлорометане и рассчитанных максимумов поглощения $\lambda_{\mathrm{abs}}^{\text {theor }}$ и флуоресценции $\lambda_{\mathrm{fl}}^{\text {theor }}$

\begin{tabular}{c|c|c|c|c|c|c|c}
\hline Краситель & $\begin{array}{c}\lambda_{\mathrm{abs}}^{\text {exp }} \\
\mathrm{nm}\end{array}$ & $\begin{array}{c}\varepsilon \cdot 10^{-4}, \\
1 \cdot \mathrm{mol}^{-1} \cdot \mathrm{cm}^{-1}\end{array}$ & $\begin{array}{c}\lambda_{\mathrm{abs}}^{\text {theor }}, \\
\mathrm{nm}\end{array}$ & $\begin{array}{c}\lambda_{\mathrm{abs}}^{\text {theor }} \\
\text { cor., } \mathrm{nm}\end{array}$ & $\begin{array}{c}\lambda_{\mathrm{fl}}^{\text {exp }}, \\
\mathrm{nm}\end{array}$ & $\begin{array}{c}\lambda_{\mathrm{fl}}^{\text {theor }}, \\
\mathrm{nm}\end{array}$ & $\begin{array}{c}\lambda_{\mathrm{fl}}^{\text {theor }} \\
\mathrm{cor} ., \mathrm{nm}\end{array}$ \\
\hline $\mathbf{1}$ & 1063 & 32.534 & 698.6 & 925.1 & 1120 & 721.3 & 951.8 \\
$\mathbf{2}$ & 1017 & 26.005 & 667.6 & 867 & 1082 & 691.7 & 912.1
\end{tabular}

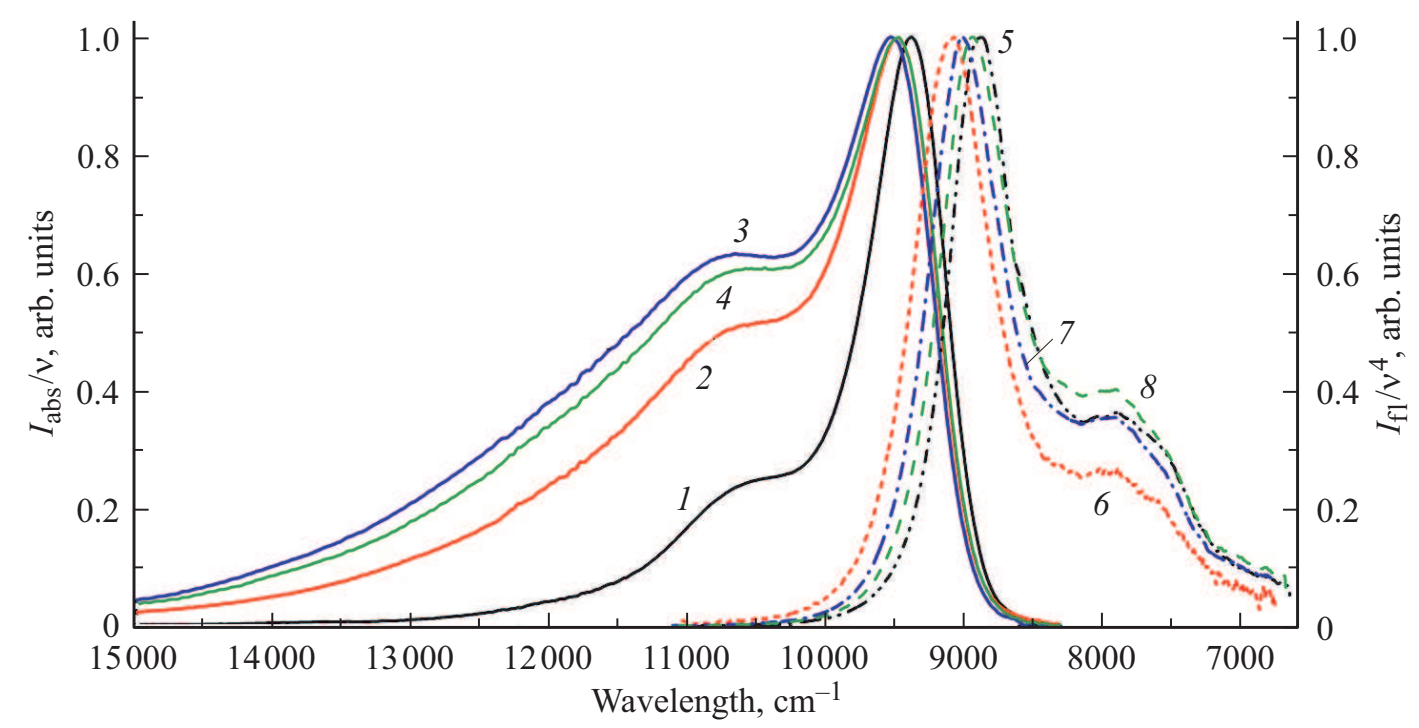

Рис. 2. Электронные спектры поглощения $(1-4)$ и флуоресценции $(5-8)$ красителя 1 в ДХМ $(1,5)$, этаноле $(2,6)$, ацетонитриле $(3,7)$, ацетоне $(4,8) . I_{\mathrm{abs}}, I_{\mathrm{fl}}$ - интенсивности поглощения и флуоресценции. Длина волны возбуждения флуоресценции $445 \mathrm{~nm}$.

Сохранение отчетливого универсального контура полосы для полиметинов, поглощающих свет в области более $1000 \mathrm{~nm}$, весьма нетривиально. Дело в том, что достижение этого практически важного спектрального диапазона осуществляется в первую очередь за счет удлинения полиметиновой цепи [2]. В случае тиопирилоцианинов 1 и 2 она достигает 7 метиновых групп. При такой длине цепи нарушается равномерность в распределении заряда в хромофоре красителя [19]. Особенно оно существенно в случае, когда его концевые группы отклоняются от средней электронодонорности [21]. Тиопирилиевые концевые группы красителей 1 и $\mathbf{2}$ обладают слабой электронодонорностью. Исходя из этого и длины полиметиновой цепи для них следует ожидать значительного нарушения равномерности распределения заряда. В свою очередь, его нарушение обусловливает усиление специфических электростатических взаимодействий между распределенным зарядом хромофора и диполями молекул растворителя. В случае катионных красителей, к которым относятся соединения 1 и 2 , главный вклад в эти взаимодействия вносит нуклеофильная сольватация положительно заряженных центров хромофора тиопирилоцианинов $[21,22]$. Это должно привести к падению селективности поглощения, сопровождающемуся уширением полосы, уменьшением интенсивности длинноволнового максимума и ее ростом у коротковолнового мак- симума. Действительно, такая картина наблюдается для большинства поликарбоцианинов, поглощающих свет в области более $1000 \mathrm{~nm}[21,22]$.

У красителей 1 и 2 также обнаруживается сильное изменение интенсивности и формы полосы поглощения при замене ДХМ более полярными и нуклеофильными растворителями, такими как ацетон, ацетонитрил и этанол (рис. 1 и 2). Показательно, что в ДХМ полоса поглощения красителя $\mathbf{1}$ уже и интенсивнее, чем таковая у 2, тогда как в ацетонитриле и других полярных растворителях все наоборот. Во всех этих растворителях соблюдается закон Ламберта-Бера в более широком интервале концентраций $10^{-6}-10^{-4} \mathrm{~mol} / \mathrm{l}$, чем рабочая концентрация $5 \cdot 10^{-6} \mathrm{~mol} / 1$ красителей 1 и 2. Это свидетельствует о том, что наблюдаемые изменения в спектрах поглощения красители 1 и 2 не связаны с их ассоциацией, а определяются эффектами сольватации. Поскольку наблюдаемые спектральные изменения аналогичны как в апротонных (ацетон, ацетонитрил), так и в протонодонорных (этанол) растворителях (рис. 2), то водородные связи не вносят в них существенный вклад. Следовательно, главную роль в сольватации играют электростатические взаимодействия распределенного заряда хромофора с диполями растворителя.

Для количественной характеристики распределения заряда в хромофоре воспользуемся величинами суммар- 
Таблица 2. Результаты квантово-химического расчета зарядов на отдельных атомах $(q)$ и суммарных зарядов на концевых группах $\Sigma q_{N}$ и в полиметиновой цепи $\Sigma q_{C}$ красителей 1 и 2 в основном $S_{0}$, франк-кондоновском первом возбужденном $S_{1}^{\mathrm{FC}}$, флуоресцентном $S_{1}$ и франк-кондоновском основном $S_{0}^{\mathrm{FC}}$ состояниях

\begin{tabular}{|c|c|c|c|c|c|c|c|c|c|c|c|c|}
\hline State & $q \mathrm{~S}$ & $q \mathrm{C} 2$ & $q \mathrm{C} 3$ & $q \mathrm{C} 4$ & $q \mathrm{C5}$ & $q \mathrm{C} 6$ & $q \mathrm{C} 7$ & $q \mathrm{C} 8$ & $q \mathrm{C} 9$ & $q \mathrm{C} 10$ & $\Sigma q_{N}$ & $\Sigma q_{C}$ \\
\hline \multicolumn{13}{|c|}{ Краситель 1} \\
\hline$S_{0}$ & 0.511 & -0.144 & 0.040 & 0.003 & 0.032 & -0.141 & -0.011 & 0.080 & -0.017 & 0.013 & 0.879 & 0.120 \\
\hline$S_{1}^{\mathrm{FC}}$ & 0.507 & -0.155 & 0.051 & -0.029 & 0.045 & -0.148 & 0.034 & 0.036 & 0.038 & -0.033 & 0.843 & 0.154 \\
\hline$S_{1}$ & 0.496 & -0.150 & 0.051 & -0.029 & 0.045 & -0.144 & 0.036 & 0.034 & 0.045 & -0.040 & 0.839 & 0.160 \\
\hline$S_{0}^{\mathrm{FC}}$ & 0.501 & -0.140 & 0.041 & 0.000 & 0.033 & -0.137 & -0.007 & 0.074 & -0.011 & 0.003 & 0.892 & 0.116 \\
\hline \multicolumn{13}{|c|}{ Краситель 2} \\
\hline$S_{0}$ & 0.504 & -0.144 & 0.039 & -0.002 & 0.029 & -0.142 & -0.014 & 0.072 & -0.016 & 0.072 & 0.850 & 0.154 \\
\hline$S_{1}^{\mathrm{FC}}$ & 0.501 & -0.156 & 0.052 & -0.035 & 0.044 & -0.149 & 0.036 & 0.029 & 0.033 & 0.027 & 0.809 & 0.188 \\
\hline$S_{1}$ & 0.491 & -0.151 & 0.052 & -0.035 & 0.045 & -0.145 & 0.038 & 0.026 & 0.036 & 0.023 & 0.809 & 0.188 \\
\hline$S_{0}^{\mathrm{FC}}$ & 0.494 & -0.140 & 0.040 & -0.005 & 0.032 & -0.139 & -0.008 & 0.065 & -0.012 & 0.065 & 0.857 & 0.150 \\
\hline
\end{tabular}

ных зарядов на атомах концевых групп $\Sigma q_{N}$ и полиметиновой цепи $\Sigma q_{C}$, введенными в работе [21].

Наиболее контрастное изменение электронной плотности у полиметиновых красителей происходит в полиметиновой цепи. Из табл. 2 видно, что в основном состоянии у тиопирилоцианинов 1 и 2 имеет место альтернация положительных и отрицательных зарядов на углеродных атомах. Чем сильнее такая альтернация, тем ближе электронное строение красителя к идеальному полиметиновому состоянию. Введение триметиленовой мостиковой группы и атома хлора в полиметиновую цепь значительно уменьшает положительный заряд в ней и увеличивает его в тиопирилиевом цикле. Это происходит, главным образом, за счет снижения заряда в мезо-положении цепи и его роста на атомах $\mathrm{S}, \mathrm{C} 3, \mathrm{C} 4$ (меняется с минуса на плюс) и С5 гетероцикла. В результате различие между величинами $\Sigma q_{N}$ и $\Sigma q_{C}$ у красителя 1 становится больше, чем у тиопирилоцианина 2 (табл. 2). Следовательно, у первого в большей степени нарушена равномерность в распределении заряда по сравнению с последним. Поэтому краситель 1 сильнее подвержен нуклеофильной сольватации, чем 2, особенно в полярных растворителях, вследствие чего вибронный контур его длинноволновой полосы существеннее искажается межмолекулярными взаимодействиями со средой (рис. 1 и 2). Эти искажения могут происходить как за счет однородного, так и неоднородного уширения полосы поглощения [21,22]. Первое вызвано усилением вибронных взаимодействий под действием среды, а последнее - образованием специфически сольватированных форм красителей. Оба механизма обусловливают падение интенсивности в длинноволновой области и ее рост в коротковолновой [21]. В ряде работ на примере высших винилогов полиметинов с сильноэлектронодонорными концевыми группами сильное уширение полос поглощения объясняется однородным механизмом уширения $[23,24]$. Авторы считают, что под действием полярных взаимодействий со средой нарушается электронная симметрия хромофора (коллапс симметрии) [23,24]. В результате типичный симметричный краситель приобретает свойства несимметричного $[25,26]$. Полярный растворитель, преимущественно сольватируя концевую группу, на которую смещается заряд, еще более увеличивает электронную асимметрию красителя. Это усиливает вибронные взаимодействия за счет увеличения вероятности переходов на высшие колебательные подуровни, что и вызывает однородное уширение [26]. Однако в случае красителей $\mathbf{1}$ и $\mathbf{2}$ перераспределение заряда в хромофоре при возбуждении, отображаемое величинами $\Sigma q_{N}$ и $\Sigma q_{C}$, не сопровождается нарушением электронной симметрии хромофора. Иными словами, заряды на симметричных атомах относительно плоскости симметрии $C_{2 v}$, проходящей через мезо-углеродный атом (C10) полиметиновой цепи перпендикулярно плоскости хромофора, оказываются равными. Расчет показывает, что эта тенденция сохраняется и в более полярном, чем ДХМ, ацетонитриле. Более того, переход от вакуума к раствору сопровождается выравниванием заряда при сохранении электронной симметрии хромофора. Так, величины $\Sigma q_{N}$ и $\Sigma q_{C}$ в вакууме и ацетонитриле соответственно равны 0.910 и $0.102,0.877$ и 0.121 в состоянии $S_{0}$ и 0.874 и $0.127,0.823$ и 0.164 в состоянии $S_{1}$. Таким образом, в случае красителей 1 и 2 коллапс симметрии, 
приводящий к неоднородному уширению полос, не должен реализоваться.

Отметим также, что нарушение электронной симметрии у полиметинов возможно за счет преимущественной локализации противоиона у одной из концевых групп [27]. Такой эффект известен в кристаллах $[28,29]$ и в малополярных растворителях с диэлектрической проницаемостью $\varepsilon_{D}<5$ в случае образования красителем контактных ионных пар [27]. В данной работе такой случай не реализуется, поскольку спектры поглощения красителей 1 и $\mathbf{2}$ не зависят от природы аниона, что обусловлено использованием растворителей с $\varepsilon_{D}>5$.

Эффект заместителей в хромофоре отражается не только в изменении электронной плотности, но и в изменении порядков связей. Это должно вносить заметный вклад в величину вибронных взаимодействий при возбуждении хромофора. Количественно оценить ее можно на основании квантово-химических расчетов величины квадратичных изменений порядков связей $\delta$ при электронном возбуждении [30]:

$$
\delta=\sqrt{\sum_{i=1}^{m}\left(P^{*}-P^{0}\right)_{i}^{2}},
$$

где $i$ - номер связи, $m$ - число связей.

Меньшая величина $\delta$ у красителя $1(\delta=0.1089)$ по сравнению с $2(\delta=0.1145)$ свидетельствует о том, что у первого вибронные взаимодействия слабее, чем у второго. Следствием этого является сужение полосы поглощения и рост интенсивности основного максимума в слабо сольватирующем ДХМ у красителя 1 по сравнению с его аналогом 2 (рис. 1). Результатом этого является существенный рост поперечного сечения поглощения на длине волны генерации $1060 \mathrm{~nm}$ $\left(12.4 \cdot 10^{16} \mathrm{~cm}^{2}\right)$ широко используемых твердотельных неодимовых лазеров [3]. Это весьма важный фактор как при использовании красителя $\mathbf{1}$ в качестве активной лазерной среды, так и пассивного затвора этих лазеров. В первом случае обеспечивается эффективная накачка длиной волны $1060 \mathrm{~nm}$, а в последнем снижается порог просветления красителя [3].

Форма полос флуоресценции (в отличие от формы полос поглощения) сохраняет универсальный контур как в малополярном ДХМ, так и во всех используемых полярных растворителях (рис. 2). При этом форма полос флуоресценции практически не зависит от природы растворителя. Несколько различается лишь положение полосы, определяемое универсальными взаимодействиями, основной вклад в которые вносит показатель преломления среды [22].

Для более объективного сравнения спектров поглощения и флуоресценции мы проанализировали их в координатах Б.И. Степанова, в которых наиболее строго соблюдается закон зеркальной симметрии Л.В. Левшина [31].
Обнаружено, что у красителей 1 и 2 в ДХМ этот закон практически выполняется, тогда как в полярных растворителях он существенно нарушается (рис. 2). Нарушение закона зеркальной симметрии в этих растворителях, как видно из рис. 2, происходит за счет меньшей чувствительности формы полос флуоресценции, чем формы полос поглощения к природе среды. Это свидетельствует о том, что в флуоресцентном состоянии $S_{1}$ красителей 1 и 2 специфические электростатические взаимодействия между зарядами их хромофора и диполями молекул растворителя ослаблены по сравнению с таковыми в основном состоянии $S_{0}$. Для понимания причины этого выполнен сравнительный анализ распределения заряда в состояниях $S_{0}$ и $S_{1}$. Отметим, что обычно при интерпретации спектров флуоресценции красителей прибегают к анализу их электронного строения во франк-кондоновском первом возбужденном состоянии $S_{1}^{\mathrm{FC}}$, что является грубым приближением, так как не учитывает геометрию релаксированного возбужденного состояния и перестройку сольватной оболочки. Поэтому для получения объективной информации об электронном строении в состояниях $S_{0}$ и $S_{1}$ квантово-химический расчет красителей 1 и 2 включал следующие этапы: 1) оптимизацию геометрии красителя в основном состоянии в поле растворителя, 2) расчет длинноволновых поглощательных переходов $S_{1}^{\mathrm{FC}} \leftarrow S_{0}$ из основного состояния в первое возбужденное фран-кондоновское $S_{1}^{\mathrm{FC}}$, которые, согласно принципу Франка-Кондона, происходят при неизменной геометрии состояния $S_{0}$ как молекулы красителя, так и молекул растворителя в сольватной оболочке (при этом, однако, учитывается быстрая составляющая релаксации растворителя, т.е. реполяризация его молекул под влиянием изменившегося распределения электронной плотности в хромофоре красителя), 3) перестройку сольватной оболочки под изменившееся электронное строение сольватируемой молекулы, 4) самосогласованную оптимизацию геометрии возбужденного состояния исследуемой молекулы и ее сольватной оболочки (образование состояния $\left.\left.S_{1}\right), 5\right)$ расчет длинноволнового флуоресцентного перехода $S_{1} \rightarrow S_{0}^{\mathrm{FC}}$ из состояния $S_{1}$ во франк-кондоновское основное состояние $S_{0}^{\mathrm{FC}}$ с учетом принципа Франка-Кондона, но уже при геометрии состояния $S_{1}$.

Из данных расчета видно, что наибольшее изменение заряда в хромофоре красителей 1 и 2 при электронном возбуждении происходит на атомах полиметиновой цепи (табл. 2). На некоторых из них знак заряда меняется на противоположный. В состоянии $S_{1}$ абсолютные величины зарядов изменяются сильнее не только по сравнению с состоянием $S_{0}$, но и с $S_{1}^{\mathrm{FC}}$. Из меньших различий между величинами $\Sigma q_{N}$ и $\Sigma q_{N}$ в флуоресцентном состоянии, чем в основном, следует, что заряды более выровнены в состоянии, названном первым. Поэтому электростатические взаимодействия катиона красителя с молекулами среды в состоянии $S_{1}$ по сравнению с $S_{0}$ должны быть ослаблены. Это обстоятельство обусловливает низкую чувствительность формы полос флуоресценции тиопи- 


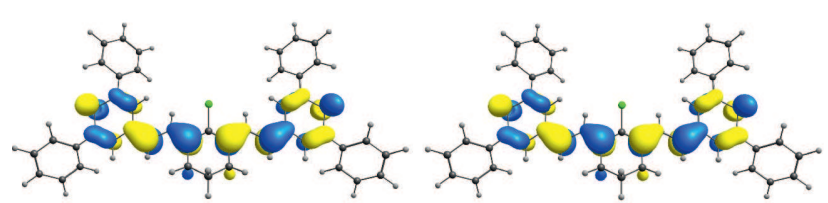

HOMO

LUMO

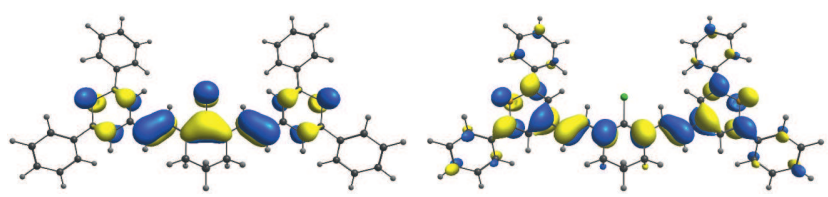

HOMO-1

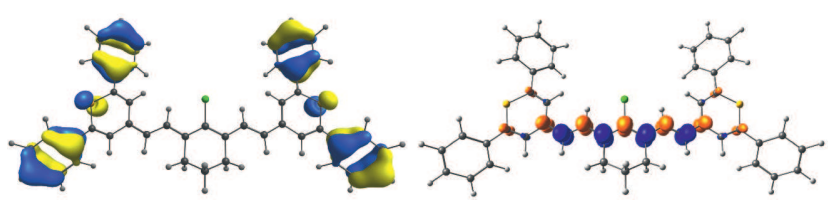

HOMO-8

Рис. 3. Форма молекулярных орбиталей и изменение электронной плотности при переходе $S_{1}^{\mathrm{FC}} \leftarrow S_{0}$ (оранжевый увеличение, синий - уменьшение).

рилоцианинов 1 и 2 к полярности среды и их резкое сужении относительно полос поглощения, приводящее к нарушению классического закона зеркальной симметрии В.Л. Левшина.

Из анализа величин $\delta$ следовало ожидать уширения полос флуоресценции за счет усиления вибронных взаимодействий в переходе $S_{1} \rightarrow S_{0}^{\mathrm{FC}}(\delta=0.1168$ и 0.1215 для красителей $\mathbf{1}$ и 2 ) по сравнению с $S_{1}^{\mathrm{FC}} \leftarrow S_{0}$ $(\delta=0.1089$ и 0.1145 для тех же красителей). Действительно, это имеет место в ДХМ, на что четко указывает большая интенсивность колебательного максимума в полосе флуоресценции, чем в полосе поглощения (рис. 2). В полярных растворителях, где эффект среды имеет доминирующее влияние на изменение формы полос поглощения, наблюдается противоположная картина.

Рассчитанные длинноволновые электронные переходы тиопирилоцианинов $\mathbf{1}$ и $\mathbf{2}$ имеют $\boldsymbol{\pi} \rightarrow \pi^{*}$-природу и происходят между одинаковыми типами орбиталей. Они несколько отличаются лишь процентным вкладом этих орбиталей. Охарактеризуем электронные переходы на примере красителя 1. Его длинноволновый поглощательный переход $S_{1}^{\mathrm{FC}} \leftarrow S_{0}$ обусловлен практически полным (97.8\%) переносом электронной плотности с НОМО на LUMO (рис. 3). При этом происходит значительное чередование увеличения и уменьшения электронной плотности на атомах полиметиновой цепи и в меньшей степени на атомах тиопирилиевых ядер (рис. 3). Расчетный максимум поглощения длинноволнового перехода с учетом поправки [16] составляет $925 \mathrm{~nm}$. Видно, что несмотря на поправку, он занижен по сравнению с экспериментом (табл. 1). По-видимому, введенная поправка неудовлетворительно работает в ближнем ИК диапазоне спектра, поскольку она получена на основании спектров поглощения видимого диапазона, к тому же небольшого числа красителей [18].

Расчет в согласии с экспериментом и правилом Ферстера-Дьюара-Нотта [2] о влиянии электронодонорных (шестизвенного цикла) и электроноакцепторных (атома хлора) заместителей соответственно в обогащенных и обедненных электронной плотностью положениях хромофора отображает углубление окраски при переходе от красителя 2 к 1 (табл. 1).

Стационарная флуоресценция красителей 1 и 2 независимо от длины волны возбуждения наблюдается только с длинноволнового перехода $S_{1} \rightarrow S_{0}^{\mathrm{FC}}$. Ее расчетный максимум с учетом поправки [18] равен $952 \mathrm{~nm}$. Его величина (так же как и величина максимума поглощения), несмотря на поправку, занижена по сравнению с экспериментом, но несколько меньше (табл. 1).

Флуоресцентный переход $S_{1} \rightarrow S_{0}^{\mathrm{FC}}$ осуществляется между теми же орбиталями, что и переход $S_{1}^{\mathrm{FC}} \leftarrow S_{0}$. Однако вклад НOMO и LUMO в первый немного выше $(98.0 \%)$, чем в последний $(97.8 \%)$. При этом несколько уменьшается роль МО, вносящих незначительный вклад: с HOMO-1 на LUMO+1 (0.95\%) и с HOMO-8 на LUMO $(0.33 \%)$ в флуоресценции и между теми же MO $0.97 \%$ и $0.34 \%$ в поглощении. Из расчетов следует, что рост вклада HOMO и LUMO в переход $S_{1} \rightarrow S_{0}^{\mathrm{FC}}$ вызван большим смещением электронной плотности с фенильных групп в полиметиновый хромофор в флуоресцентном состоянии по сравнению с основным у тиопирилотрикарбоцианинов $\mathbf{1}$ и 2. Дело в том, что в состоянии $S_{0}$ эти группы, как следует из расчета, выведены из плоскости хромофора на $37^{\circ}$ и $38^{\circ}$ за счет стерических препятствий с объемистым атомом серы (рис. 4,a). При электронном возбуждении длина связи C-S несколько удлиняется (в состоянии $S_{0}$ и $S_{1}$ длина связи C2-S равна 0.17535 и $0.17598 \mathrm{~nm}$, а в $\mathrm{C} 6-\mathrm{S}-0.17540$ и $0.17592 \mathrm{~nm}$ соответственно), что уменьшает упомянутые выше препятствия, следствием чего является частичное уплощение фенильной группы. Так, угол поворота $\mathrm{Ph}$ у красителя 1 в флуоресцентном состоянии уменьшается при атоме С2 до $33^{\circ}$, а при атоме С6 - до $35^{\circ}$ (рис. 4, $b$ ). Это приводит к росту порядка связей при переходе $S_{1} \leftarrow S_{0}$ с 1.0674 до $1.0839 \mathrm{~nm}$ и с 1.0697 до $1.0899 \mathrm{~nm}$ для С2-Ph и С6-Ph соответственно. В результате увеличивается сопряжение фенильной группы с основным хромофором, что в свою очередь вызывает дополнительный батохромный сдвиг полосы флуоресценции по сравнению с полосой поглощения. За счет этого возрастает стоксов сдвиг.

Основным каналом безызлучательной дезактивации возбужденного состояния полиметиновых красителей считается фотоизомеризация вокруг связей полиметиновой цепи в этом состоянии [1,32]. Однако причина усиления изомеризации при фотовозбуждении однозначно не сформулирована. Для выяснения этой причины мы сравнили изменения порядков связей в четырех состояниях. 
Таблица 3. Результаты квантово-химических расчетов порядков связей в полиметиновой цепи и величины $\Delta P$

\begin{tabular}{l|c|c|c|c|c}
\hline State & $\begin{array}{c}\text { Bond } \\
4-7\end{array}$ & $\begin{array}{c}\text { Bond } \\
7-8\end{array}$ & $\begin{array}{c}\text { Bond } \\
8-9\end{array}$ & $\begin{array}{c}\text { Bond } \\
9-10\end{array}$ & $\begin{array}{c}\Delta P \\
\text { Chain }\end{array}$ \\
\hline \multicolumn{6}{c}{ Краситель 1 } \\
\hline$S_{0}$ & 1.3346 & 1.4166 & 1.3710 & 1.3506 & 0.0256 \\
$S_{1}^{\mathrm{FC}}$ & 1.2864 & 1.4294 & 1.3322 & 1.3330 & 0.0421 \\
$S_{1}$ & 1.2652 & 1.4533 & 1.3172 & 1.3385 & 0.0549 \\
$S_{0}^{\mathrm{FC}}$ & 1.3164 & 1.4345 & 1.3589 & 1.3540 & 0.0343 \\
\hline \multicolumn{5}{|c}{ Краситель 2 } \\
\hline$S_{0}$ & 1.3480 & 1.4080 & 1.4097 & 1.4033 & 0.0221 \\
$S_{1}^{\mathrm{FC}}$ & 1.2950 & 1.4182 & 1.3712 & 1.3904 & 0.0369 \\
$S_{1}$ & 1.2723 & 1.4419 & 1.3574 & 1.3964 & 0.0522 \\
$S_{0}^{\mathrm{FC}}$ & 1.3278 & 1.4263 & 1.3981 & 1.4078 & 0.0311
\end{tabular}

Для количественной оценки альтернации порядков связей воспользуемся введенной в работе [33] величиной $\Delta P$ :

$$
\Delta P=1 / m \sum_{i=1}^{m}\left|P_{a v}-P_{i}\right|,
$$

где $P_{a v}=1 / m \sum_{i=1}^{m} P_{i}$ (средний порядок связи), $P_{i}-$ порядок $i$-й связи, $m$ - число связей.

Из данных квантово-химического расчета следует, что как у красителя $\mathbf{1}$, так и $\mathbf{2}$ величина $\Delta P$ значительно больше в состояниях $S_{1}^{\mathrm{FC}}$ и $S_{1}$, чем в состоянии $S_{0}$ (табл. 3). Это указывает на далеко неочевидный факт, что у первых альтернация простых и двойных связей сильнее, чем у последнего. Иными словами, порядок связей, близких к простым, понижается, а к двойным повышается при возбуждении. Это снижает барьер вращения вокруг формально простых связей, что и увеличивает вероятность фотоизомеризации в состоянии $S_{1}$ и усиливает вибронные взаимодействия, как отмечалось выше, в переходе $S_{1} \rightarrow S_{0}^{\mathrm{FC}}$ по сравнению с переходом $S_{1}^{\mathrm{FC}} \leftarrow S_{0}$.
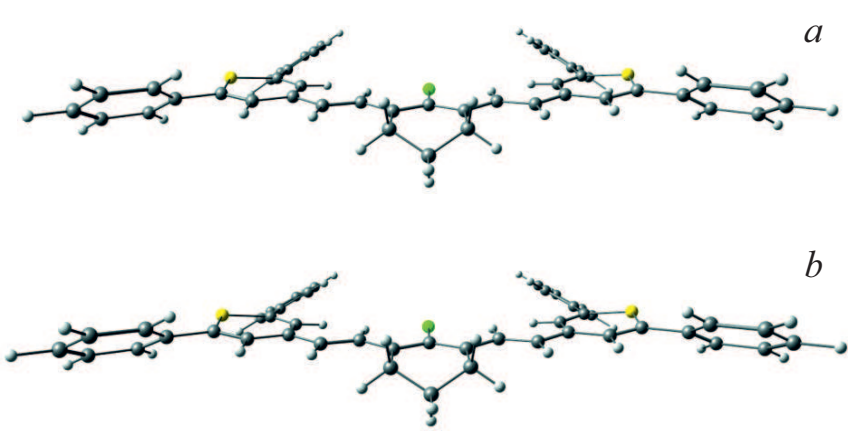

Рис. 4. (a) Угол поворота фенила $37.4^{\circ}$ и $38^{\circ}$ вокруг связей $\mathrm{C} 2-\mathrm{Ph}$ и $\mathrm{C} 6-\mathrm{Ph}$ соответственно у красителя 1 в состоянии $S_{0}$; (b) угол поворота фенила $33.3^{\circ}$ и $35.2^{\circ}$ вокруг связей $\mathrm{C} 2-\mathrm{Ph}$ и $\mathrm{C} 6-\mathrm{Ph}$ соответственно у красителя 1 в состоянии $S_{1}$.
Показательно, что циклическая группировка в $\gamma, \gamma^{\prime}$-положениях тиопирилотрикарбоцианина 1 усиливает альтернацию порядков связей в цепи по сравнению с его незамещенным аналогом 2 (табл. 2). Отсюда можно ожидать, что триметиленовая мостиковая группировка вопреки традиционным представлениям может не только повышать квантовый выход флуоресценции за счет увеличения жесткости хромофора, но и понижать его в результате облегчения фотоизомеризации вокруг связей с пониженным порядком. Какой из этих эффектов будет доминирующим, определяется формой конкретного пикосекундного возбуждения.

У красителей 1 и $\mathbf{2}$ из-за различия в строении полиметиновой цепи квантовые выходы $\varphi$ должны существенно отличаться. Поэтому для объективного сравнения их квантовых выходов флуоресценции мы определили $\varphi$ в полимерных пленках, где процессы фотоизомеризации сведены к минимуму. Значения $\varphi$ рассчитаны из данных времен жизни состояния $S_{1}^{\mathrm{FC}}$ красителей, полученных методом пикосекундного флеш-фотолиза (возбуждениязондирования) [34]. Важно отметить, что квантовый выход флуоресценции красителя $1(0.76 \%)$ выше, чем у $2(0.57 \%)$, несмотря на то, что он поглощает свет в более критичном, с точки зрения энергетического интервала, длинноволновом ближнем ИК диапазоне спектра. Вероятной причиной увеличения его квантового выхода по сравнению с 2 может быть ослабление вибронных взаимодействий, как следует из значений величин $\delta$ (табл. 2).

Фенильные группы тиопирилоцианинов 1 и 2, находясь под углом к плоскости хромофора, способны в значительной степени стерически препятствовать образованию ассоциатов, которые существенно ухудшают спектрально-люминесцентные и нелинейно-оптические свойства красителей $[3,35]$. Это обстоятельство играет весьма важную роль в полимерных матрицах, особенно в тонких пленках, которые широко используются для пассивной синхронизации мод пикосекундных лазеров [3]. В таких пленках концентрация красителя на порядки превышает таковую при измерении оптических спектров в $1 \mathrm{~cm}$ кювете. При столь больших концентрациях в пленках образуются контактные ионные пары, которые за счет электростатических взаимодействий разноименных зарядов противоионов также содействуют ассоциации красителей [36]. Обычно полиметины образуют ассоциаты сэндвичего строения (Н-агрегаты) [35,36]. Поскольку такие агрегаты не люминесцируют, то они поглощенную световую энергию превращают в тепловую, вызывая локальное нагревание среды, приводящее к разрушению красителя [3]. Кроме того, образование ассоциатов полиметинов сопровождается перекрыванием их полос поглощения с аналогичными полосами соответствующих мономерных красителей. Это приводит к поглощению лазерного излучения (в случае тиопирилоцианинов 1 и $2 \lambda=1060 \mathrm{~nm}$ ) образовавшимися агрегатами в полимерных пассивных затворах, и, следовательно, к уменьшению степени просветления этих 


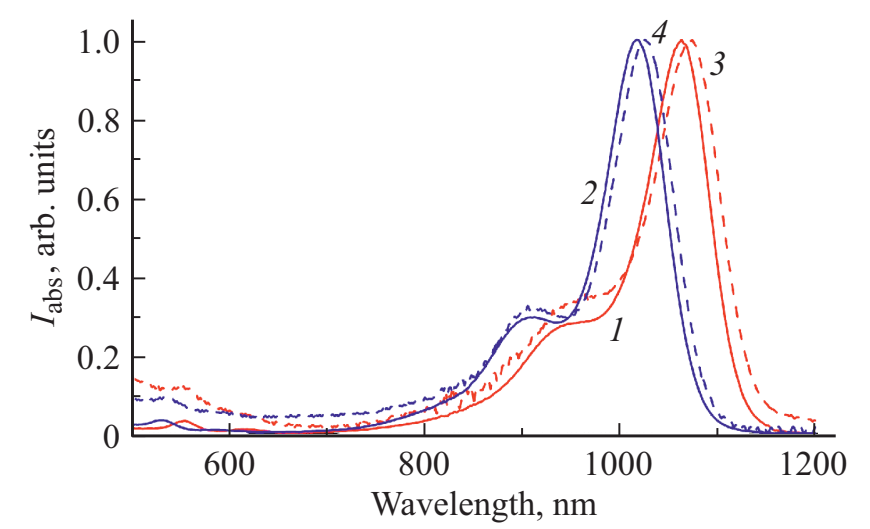

Рис. 5. Нормированные электронные спектры поглощения красителей $1(1,3)$ и $2(2,4)$ в дихлорометане и пленке поливинилэтилаля.

затворов и энергии генерируемых ими ультразвуковых импульсов [3]. Одним из методов подавления агрегации красителей является введение объемистых заместителей в их молекулы. Именно такую функцию выполняют фенильные заместители у тиопирилоцианинов 1 и 2 , а также атом хлора в мезо-положении красителя 1. Действительно, спектр поглощения тиопирилоцианинов $\mathbf{1}$ и 2 в пленке поливинилэтилаля имеет такой же контур полосы, как и в ДХМ (рис. 5).

\section{Заключение}

Краситель 1 (IR 1061) в ДХМ обладает одним из самых высоких сечений поглощения на длине волны $1060 \mathrm{~nm}$ среди органических красителей. Полоса этого перехода обладает высокой селективностью поглощения, вследствие чего IR 1061 весьма прозрачен в области 800-900 nm. На эту область разработаны эффективные светодиодные излучатели для накачки твердотельных неодимовых лазеров. Можно ожидать, что краситель 1 и его аналоги будут перспективными в качестве активных и пассивных рабочих сред лазеров, излучающих в области $1060 \mathrm{~nm}$, использующих диодную накачку в диапазоне 800-900 nm. Правда, в полярных растворителях из-за резкого падения интенсивности и селективности поглощения длинноволновой полосы (рис. 2) они мало пригодны для этих целей. Однако в этом и нет особой необходимости, поскольку жидкостные среды сегодня практически полностью вытеснены полимерными средами по причине ряда эксплуатационных преимуществ последних над первыми [37]. В полимерах же типа поливинилэтилаля краситель $\mathbf{1}$ сохраняет все спектральные достоинства, которыми он обладает в ДХМ. Поэтому полимерные как активные, так и пассивные среды на основе IR 1061 перспективны для твердотельных неодимовых лазеров с диодной накачкой.

\section{Финансирование работы}

Данная работа выполнена в рамках совместного научно-исследовательского проекта НАН Украины (проект 02-03-20) и НАН Беларуси (проект № Ф20УКА-010), финансируемого НАН Украины и НАН Беларуси.

\section{Конфликт интересов}

Авторы заявляют, что у них нет конфликта интересов.

\section{Список литературы}

[1] Ishchenko A.A. // Russ. Chem. Rev. 1991. V. 60. N 8. P. 865. doi 10.1070/rc1991v060n08abeh001116

[2] Tolmachev A.I., Slominskii Yu.L., Ishchenko A.A. // New Infrared Dyes for High Technology Applications. NATO ASI Series. 3. High Technology. / Ed. by Daehne S., Resch-Genger U., Wolfbeis O.S. Dordrecht-Boston-London: Kluwer Academic Publishers, 1998. V. 52. P. 385. doi 10.1007/978-94-011-5102-3_19

[3] Ishchenko A.A. // Quantum Electron. 1994. V. 24. N 6. P. 471. doi 10.1070/qe1994v024n06abeh000122.

[4] Mustroph H. // Phys. Sci. Rev. 2020. V. 5. 20190145. P. 1. doi 10.1515/psr-2019-0145

[5] Zhu S., Tian R., Antaris A.L., Chen X., Dai H. // Adv. Mater. 2019. V. 31. P. 1900321(1-25). doi 10.1002/adma.201900321

[6] Voiciuk V., Redeckas K., Derevyanko N.A., Kulinich A.V., Barkauskas M., Vengris M., Sirutkaitis V., Ishchenko A.A. // Dyes Pigm. 2014. V. 109. P. 120. doi 10.1016/j.dyepig.2014.05.012

[7] Bezrodnyi V.I., Derevyanko N.A., Ishchenko A.A., Kropachev A.V. // Quantum Electron. 2009. V. 39. N 1. P. 79. doi 10.1070/qe2009v039n01abeh013832

[8] Bezrodnyi V.I., Ishchenko A.A. // Appl. Phys. B. 2001. V. 73. N 3. P. 283. doi $10.1007 / \mathrm{s} 003400100646$

[9] Bondar M.V., Derevyanko N.A., Dyadyusha G.G., Zubarovskii V.M., Ishchenko A.A., Przhonskaya O.V., Slominskii Y.L., Smirnova A.L., Tikhonov E.A., Tolmachev A.I. // Sov. J. Quantum Electron. 1984. V. 14. P. 317. doi 10.1070/QE1984v014n03ABEH004888

[10] Svetlichnyi V.A., Ishchenko A.A., Vaitulevich E.A., Derevyanko N.A., Kulinich A.V. // Opt. Commun. 2008. V. 281. P. 6072. doi 10.1016/j.optcom.2008.09.067

[11] Casalboni M., De Matteis F., Prosposito P., Quatela A., Sarcinelli F. // Chem. Phys. Lett. 2003. V. 373. P. 372. doi 10.1016/S0009-2614(03)00608-0

[12] Hoshi R., Suzuki K., Hasebe N., Yoshihara T., Tobita S. // Analytical Chemistry. 2020. V. 92. N 1. P. 607. doi 10.1021/acs.analchem.9b03297

[13] Kudinova M.A., Derevyanko N.A., Dyadyusha G.G., Ishchenko A.A., Tolmachev A.I. // Chem. Heterocycl. Compd. 1980. V. 16. P. 691. doi 10.1007/BF00557737

[14] Gaussian 09, Revision B.01. Frisch M.J., Trucks G.W., Schlegel H.B., Scuseria G.E., Robb M.A., Cheeseman J.R., Scalmani G., Barone V., Mennucci B., Petersson G.A., Nakatsuji H., Caricato M., Li X., Hratchian H.P., Izmaylov A.F., Bloino J., Zheng G., Sonnenberg J.L., Hada M., Ehara M., Toyota K., Fukuda R., Hasegawa J., Ishida M., Nakajima T., Honda Y., Kitao O., Nakai H., Vreven T., 
Montgomery Jr. J.A., Peralta J.E., Ogliaro F., Bearpark M., Heyd J.J., Brothers E., Kudin K.N., Staroverov V.N., Keith T., Kobayashi R., Normand J., Raghavachari K., Rendell A., Burant J.C., Iyengar S.S., Tomasi J., Cossi M., Rega N., Millam J.M., Klene M., Knox J.E., Cross J.B., Bakken V., Adamo C., Jaramillo J., Gomperts R., Stratmann R.E., Yazyev O., Austin A.J., Cammi R., Pomelli C., Ochterski J.W., Martin R.L., Morokuma K., Zakrzewski V.G., Voth G.A., Salvador P., Dannenberg J.J., Dapprich S., Daniels A.D., Farkas O., Foresman J.B., Ortiz J.V., Cioslowski J., Fox D.J. Gaussian Inc., Wallingford CT, 2010. [Электронный pecypc]

[15] Kulinich A.V., Derevyanko N.A., Ishchenko A.A., Gusyak N.B., Kobasa I.M., Romańczyk P.P., Kurek S.S. // Dyes Pigm. 2019. V. 161. P. 24. doi 10.1016/j.dyepig.2018.09.031

[16] Tomasi J., Mennucci B., Cammi R. // Chem. Rev. 2005. V. 105. P. 2999. doi $10.1021 /$ cr9904009

[17] Fabian J. // Dyes Pigm. 2010. V. 84. P. 36. doi 10.1016/j.dyepig.2009.06.008

[18] Champagne B., Guillaume M., Zutterman F. // Chem. Phys. Lett. 2006. V. 425. P. 105. doi 10.1016/j.cplett.2006.05.009

[19] Reed A.E., Weinstock R.B., Weinhold F. // J. Chem. Phys. 1985. V. 83. P. 735. doi $10.1063 / 1.449486$

[20] Liu Z., Lu T., Chen Q. // Carbon. 2020. V. 165. P. 461. doi 10.1016/j.carbon.2020.05.023

[21] Ishchenko A.A., Derevyanko N.A., Zubarovskii V.M., Tolmachev A.I. // Theor. Exp. Chem. 1984. V. 20. N 4. P. 415. doi 10.1007/BF00516576

[22] Derevyanko N.A., Dyadyusha G.G., Ishchenko A.A., Tolmachev A.I. // Theor. Exp. Chem. 1983. V. 19. N 2. P. 150. doi $10.1007 / \mathrm{BF} 00522419$

[23] Masunov A.E., Anderson D., Freidzon A.Ya., Bagaturyants A.A. // J. Phys. Chem. A. 2015. V. 119. N 26. P. 6807. doi 10.1021/acs.jpca.5b03877

[24] Tolbert L.M., Zhao X. // J. Amer. Chem. Soc. 1997. V. 119. N 14. P. 3253. doi $10.1021 /$ ja 9626953

[25] Tatikolov A.S., Derevyanko N.A., Ishchenko A.A., Baraldi I., Caselli M., Momicchioli F., Ponterini G. // Ber. Bunsenges. Phys. Chem. 1995. V. 99. N 5. P. 763-769. https://doi.org/10.1002/bbpc.19950990512

[26] Ishchenko A.A., Derevyanko N.A., Svidro V.A. // Dyes Pigm. 1992. V. 19. N 3. P. 169. doi 10.1016/0143-7208(92)80023-G

[27] Komarov I.V., Turov A.V., Ishchenko A.A., Derevyanko N.A., Kornilov M.Yu. // Doklady Akademii Nauk SSSR. 1989. V. 306. N 5. P. 1134.

[28] Bouit P.-A., Aronica C., Toupet L., Le Guennic B., Andraud C., Maury O. // J. Am. Chem. Soc. 2010. V. 132. N 12. P. 4328. doi $10.1021 / \mathrm{ja} 9100886$

[29] Eskandari M., Roldao J.C., Cerezo J., Miliaán-Medina B., Gierschner J. // J. Am. Chem. Soc. 2020. V. 142. N 6. P. 2835. doi 10.1021/jacs.9b10686

[30] Ishchenko A.A. // Opt. Spectrosc. 1994. V. 77. N 5. P. 691.

[31] Stepanov B.I., Kazachenko L.P. // J. Appl. Spectrosc. 1971. V. 14. P. 596. doi 10.1007/BF00605796

[32] Kaliteevskaya E.N., Razumova T.K., Tarnovskii A.N. // Opt. Spectrosc. 1999. V. 86. N 1. P. 126.

[33] Ishchenko A.A., Svidro V.A., Derevyanko N.A., Slominsky Yu.L., Tolmachev A.I. // Opt. Spectrosc. 1989. V. 66. N 6. P. 1302.

[34] Демчук М.И., Михайлов В.П., Павлович В.С., Юмашев К.В., Ищенко А.А., Еремеева Е.П., Смирнова З.А., Толмачев А.И. // Химическая физика. 1986. Т. 5. № 9. C. 1184.
[35] Ishchenko A.A., Dokukina A.F., Smirnova Z.A., Tolmachev A.I. // Doklady Akademii Nauk SSSR. 1985. V. 284. N 6. P. 1407.

[36] Ishchenko A.A., Kramarenko F.G., Maydannic A.G., Sereda S.V., Vasilenko N.P. // J. Inf. Rec. Mater. 1991. V. 19. N 3. P. 207.

[37] Bezrodnyi V.I., Ishchenko A.A. // Optics and Laser Technology. 2002. V. 34. N 1. P. 7. doi 10.1016/s0030-3992(01)00080-9 